\title{
Hadronic decays of the omega meson measured with WASA-at-COSY
}

\author{
Lena HEIJKENSKJÖLD*† \\ Department of Physics and Astronomy, Uppsala University, \\ Lägerhyddsvägen 1, Uppsala, Sweden \\ E-mail: lena.heijkenskjoldephysics.uu.se
}

In this article ongoing studies of the decays $\omega \rightarrow \pi^{+} \pi^{-} \pi^{0}$ and $\omega \rightarrow \pi^{+} \pi^{-}$will be presented. The data was collected by the WASA-at-COSY collaboration where the $\omega$ mesons were produced through the reaction $p+d \rightarrow{ }^{3} \mathrm{He}+\omega$. The experiment allows for tagging of the recoil ${ }^{3} \mathrm{He}$ and a full reconstruction of all decay particles.

The isospin breaking $\omega \rightarrow \pi^{+} \pi^{-}$decay can give insight into the behaviour of the $\rho-\omega$ mixing. This channel has been studied in $e^{+} e^{-}$collisions where the interference has been conclusively shown as destructive. Only a few measurements with limited statistics exist for hadronic production of the $\omega$ meson which hint at a possible constructive interference. The aim of the present study is to investigate the structure of the $\omega \rightarrow \pi^{+} \pi^{-}$signal in proton deuteron collisions.

The decay mechanism of the $\omega \rightarrow \pi^{+} \pi^{-} \pi^{0}$ channel can be studied through a Dalitz plot in the three pion system. The aim of the study presented here is to produce an experimental Dalitz plot, which will provide test of theoretical predictions and in particular will increase understanding of three pion final state interactions.

53rd International Winter Meeting on Nuclear Physics

26-30 January 2015

Bormio, Italy

${ }^{*}$ Speaker.

${ }^{\dagger}$ for the WASA-at-COSY collaboration 


\section{WASA-at-COSY}

WASA-at-COSY is an international collaboration consisting of 36 institutes from 8 different countries. The WASA experiment has been located at the COSY ring in Forschungszentrum Jülich in Germany where it performed its last experiment in 2014 before decommissioning. The available beam from the COSY facility and the design of the WASA detector was optimised for the study of interactions and decays of light mesons formed in collisions of protons and deuterons. The light mesons include $\pi^{0}, \eta$ and $\omega$ and studies of the decays of these mesons are important for investigations of fundamental physics of the medium energy range. This article will focus on two $\omega$ meson decay channels using data from proton-deuteron interactions collected at the WASA detector.

\subsection{The $\omega$ program}

For the experiments focused on producing the $\omega$ meson, two complementary production reactions were used, $p p \rightarrow p p \omega$ and $p d \rightarrow{ }^{3} \mathrm{He} \omega$. The collected data sets are currently studied with the focus on the three most common decay channels and a exploratory search for the decay channel which would allow measurements of the $\omega \rightarrow \pi^{0} \gamma *$ transition form factor, all listed in Table 1.

\begin{tabular}{|l|l|l|}
\hline Decay & Branching ratio & Interesting physics \\
\hline$\omega \rightarrow \pi^{+} \pi^{-} \pi^{0}$ & $89.2 \times 10^{-2}$ & Dalitz plot parameters \\
\hline$\omega \rightarrow \pi^{0} \gamma$ & $8.28 \times 10^{-2}$ & Branching ratio \\
\hline$\omega \rightarrow \pi^{+} \pi^{-}$ & $1.53 \times 10^{-2}$ & $\rho-\omega$ interference \\
\hline$\omega \rightarrow \pi^{0} e^{+} e^{-}$ & $7.7 \times 10^{-4}$ & Transition form factor \\
\hline
\end{tabular}

Table 1: The table lists the $\omega$ decay channels which are being studied by the WASA-at-COSY collaboration. Values of branching ratio from [1].

In this article we present the status of the $\omega \rightarrow \pi^{+} \pi^{-}$and $\omega \rightarrow \pi^{+} \pi^{-} \pi^{0}$ studies performed on data produced through the $p d \rightarrow{ }^{3} \mathrm{He} \omega$ reaction using two different kinetic energies of the proton beam, $\mathrm{T}_{\text {beam }}=1.45 \mathrm{GeV}$ and $\mathrm{T}_{\text {beam }}=1.5 \mathrm{GeV}$.

2. $\omega \rightarrow \pi^{+} \pi^{-}$

This is a G-parity violating decay which is not allowed by the strong interaction. In literature this G-parity violation has often been ascribed to a mixing of isoscalar and isovector states corresponding to the $\omega$ and $\rho$ mesons [2]. This mixing is then attributed to be the cause of the interference pattern which has been visible at the position of the omega mass in various experimental $\pi^{+} \pi^{-}$mass distributions. But how this interference manifest depends not only on the $\rho$ - $\omega$ mixing but also on the production processes of the $\pi^{+} \pi^{-}$final state [3].

Invariant $\pi^{+} \pi^{-}$mass distributions produced in $e^{+} e^{-}$collisions experiments have conclusively shown a destructive interference occurring at the $\omega$ mass position, e.g. [4]. The production amplitudes in these reactions are purely electromagnetic and are therefore easily calculable from QED.

For hadronic production on the other hand it seems the resulting interference depends on the hadronic production process. A few pion on proton experiments reported either a destructive interference [5] between the decay amplitudes or constructive interference [6]. 
Since the WASA detector has collected a large set of data using hadronic production reaction we intend to search our data for any sign of the $\rho$ - $\omega$ intereference.

The WASA detector allows to fully reconstruct all the final state particles, in this case ${ }^{3} \mathrm{He}, \pi^{+}$ and $\pi^{-}$. The signal search includes strict cuts on $I M\left(\pi^{+} \pi^{-}\right)$and a constrained fit imposing that energy and momentum is conserved for the initial and final states in the reaction $p d \rightarrow{ }^{3} \mathrm{He} \pi^{+} \pi^{-}$. The resulting $\pi^{+} \pi^{-}$mass distributions, here given as $M_{\text {missing }}=\left|P_{p}+P_{d}-P_{{ }^{H}}\right|$, is shown in Figure 1. The shaded areas in the figures are the representation of the amount of signal events as expected from the branching ratio (Table 1), without any interference. This expected number is normalised using the number of $\omega \rightarrow \pi^{+} \pi^{-} \pi^{0}$ events found in data corrected by analysis efficiencies for both channels. In our preliminary data we see no clear structure at the position of the $\omega$ mass, which

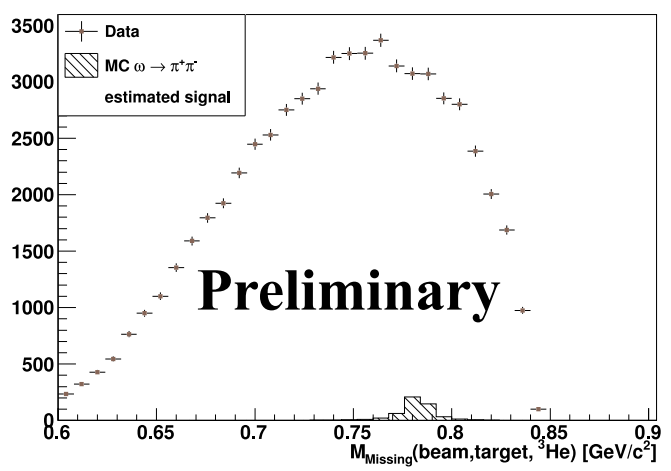

(a) $\mathrm{T}_{\text {beam }}=1.45 \mathrm{GeV}$

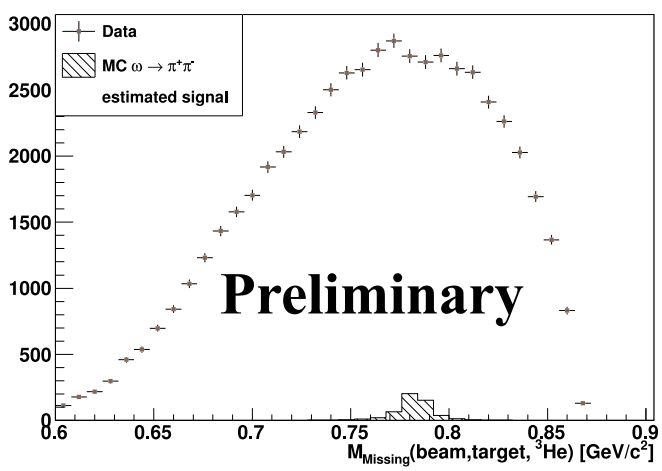

(b) $\mathrm{T}_{\text {beam }}=1.5 \mathrm{GeV}$

Figure 1: The figures show the preliminary $M_{\text {missing }}$ distributions after the full analysis aimed to collect $\pi^{+} \pi^{-}$final states. Two different energies of the proton beam was used in the experiment and the displayed distributions are not corrected for analysis efficiencies. The shaded areas illustrates the amount of $\omega \rightarrow$ $\pi^{+} \pi^{-}$events expected in these data sets.

makes the constructive interference in our production channel unlikely. By choosing a method of parametrising the $\rho-\omega$ interference, the experimental distributions could be used to extract limits on the interference parameters.

Auxiliary information to be extracted from these distributions is the isospin composition of the $\pi^{+} \pi^{-}$state when produced at our two energies. For a pair of charged pions we can only have even-even or odd-odd combinations of isopspin and relative orbital momentum. Since the latter state carries the quantum numbers of the $\rho$ meson it is reasonable to assume this resonance to be the main production channel for the odd-odd state. For a pure isoscalar distribution we may use the $p d \rightarrow{ }^{3} \mathrm{He} \pi^{0} \pi^{0}$ channel where the $\pi^{0} \pi^{0}$ can only be in an isoscalar state. This channel will tell us about the isoscalar $\pi \pi$ mass distribution at our two reaction energies which in turn will allow us to test the relative contribution of isoscalar and isovector components of the $\pi^{+} \pi^{-}$distribution. 
3. $\omega \rightarrow \pi^{+} \pi^{-} \pi^{0}$

The aim of this study is to investigate the dynamics of the $\omega \rightarrow \pi^{+} \pi^{-} \pi^{0}$ decay using Dalitz plot for the three pion system. The normalised Dalitz plot variables, $X$ and $Y$, are defined as follows, $X=\sqrt{3} \frac{T_{1}-T_{2}}{Q}$ and $Y=\frac{\left(m_{1}+m_{2}+m_{3}\right) T_{3}}{m_{1} Q}-1$, where $T_{i}$ is kinetic energy of the pion $i$ in rest frame of the decaying $\omega$ and $Q=\sum T_{i}$. Because of the symmetries of the $\omega \rightarrow \pi^{+} \pi^{-} \pi^{0}$ Dalitz plot it is convenient to describe it using the polar coordinates, $Z=X^{2}+Y^{2}$ and $\Phi=\tan ^{-1}\left(\frac{X}{Y}\right)$.

The fact that the final pions will predominantly be in a P-wave state is well known since it was used to establish the quantum numbers of the $\omega$ meson [7]. Any $\pi \pi$ pair in the final state may couple to a $\rho$ meson, but the $\pi \pi$ phase space is too small to include the full resonance. Therefore only the tail would be visible as an onset in the Dalitz plot distribution towards the edges of phase space. This onset has not been experimentally established yet. Finally there is the possibility of final-state interactions in the form of $\pi-\pi$ rescattering.

The dynamics of this decay has been calculated by several theoretical approaches, either using dispersion theory $[8,9]$ or by effective field theory calculations including vector mesons [10]. All these have produced full predictions of the density distribution in the Dalitz plot for the three pion system. It is therefore highly interesting to produce an experimental Dalitz plot where the extent of these effects can be tested.

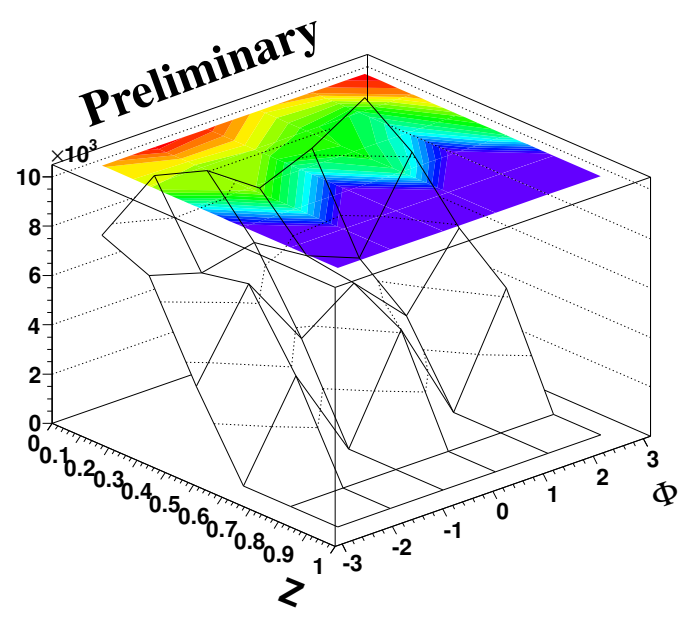

(a) $\mathrm{T}_{\text {beam }}=1.45 \mathrm{GeV}$

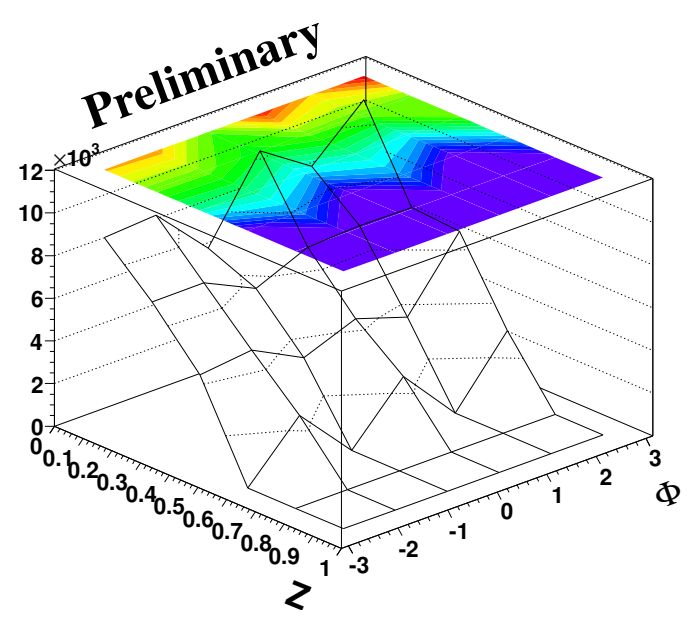

(b) $\mathrm{T}_{\text {beam }}=1.5 \mathrm{GeV}$

Figure 2: The figures show the preliminary Dalitz plot distributions after bin-wise correction for analysis efficiency. Two different energies of the proton beam was used in the experiment.

Signal selection is straightforward for the $\omega \rightarrow \pi^{+} \pi^{-} \pi^{0}$ channel, since this is the largest decay channel. After a full analysis of the data collected at both beam energies, the number of signal events is found to be 24200(600) in total. Each bin in the Dalitz plot is filled from the content of the $\omega$-peak in the corresponding $M_{\text {missing }}$ distribution. The content of each Dalitz plot bin is also corrected for the analysis efficiency of the specific bin. Shown in Figure 2 are the two resulting Dalitz plot distributions.

One way to compare our experimental Dalitz plot to theoretical predictions is to parametrise the distributions and compare the values of the parameters. Such a parametrisation can be done 
using the function

$$
F(Z, \Phi)=\mathscr{P} \cdot\left\{1+2 \alpha Z+2 \beta Z^{3 / 2} \sin 3 \Phi+2 \gamma Z^{2}+\mathscr{O}\left(Z^{5 / 2}\right)\right\},
$$

where a fixed factor, $\mathscr{P}$, incorporates the a priori known P-wave shape of the distribution and any structure on top would be parametrised by the $Z \Phi$-polynomial. To extract values of $\alpha, \beta$, $\gamma$, etc (the Dalitz plot parameters), this function is fitted to the Dalitz plots by comparing the content of each bin to the integral over bin area of the parametrisation function.

The aim of this study is to provide the two experimental Dalitz plot distributions and fitted values of the Dalitz plot parameters to the degree which we are sensitive, which is mainly limited by our statistics. The plots in Figure 3 shows the initial steps to comparing our preliminary data distributions to the shape of the Dalitz plot given by the P-wave phase space. The dynamical effects on top of the P-wave phase space are not expected by theory to be very large. Using our two data distributions combined we get preliminary indications of a small deviation between predicted phase space shape and our experimental distributions.

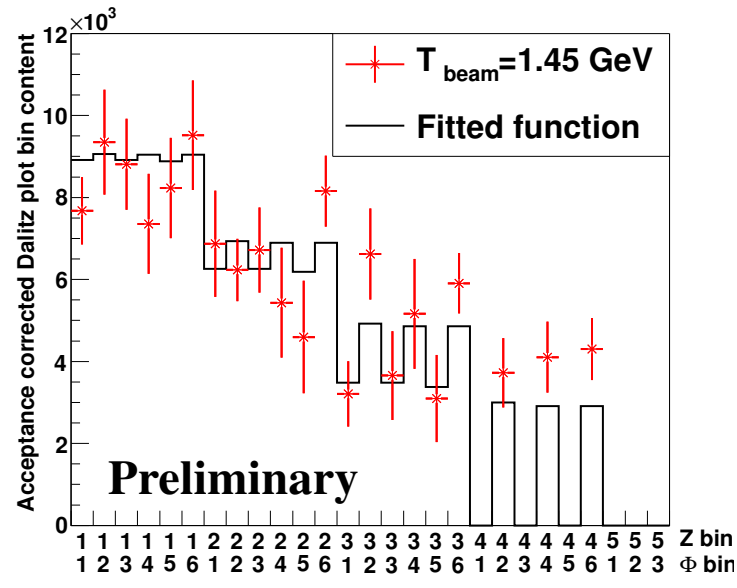

(a) $\mathrm{T}_{\text {beam }}=1.45 \mathrm{GeV}$

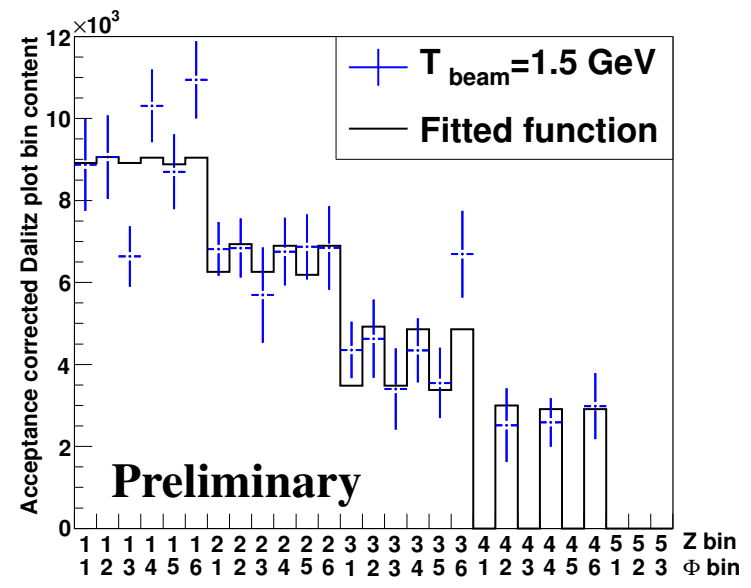

(b) $\mathrm{T}_{\text {beam }}=1.5 \mathrm{GeV}$

Figure 3: The points in the figures show the preliminary Dalitz plot distributions in one dimensional representation (only with statistical errors). The black lines shows the shape of the P-wave phase space fitted to the data distributions.

\section{References}

[1] K.A. Olive et al. (Particle Data Group), Review of particle physics, Chin.Phys. C38 (2014) 090001

[2] H. B. O'Connell et al., Rho-Omega mixing, vector meson dominance and the Pion form-factor, Prog.Part.Nucl.Phys. 39 (1997) 201-252

[3] S. M. Flatté, Decay properties of the omega meson, Phys.Rev. 145 (1966) 1050-1061

[4] CMD-2 collaboration, Measurement of $e^{+} e^{-} \rightarrow \pi^{+} \pi^{-}$cross section with CMD-2 around $\rho$-meson, Phys.Lett. B527 (2002) 161-172 [hep-ex/0112031]

[5] G. Goldhaber et al., $\omega \rho^{0}$ interference effect in the $\pi^{+} p \rightarrow \pi^{+} \pi^{-} \Delta^{++}$interaction, Phys.Rev.Lett 23 (1969) 1351 
[6] B. N. Ratcliff et al., Measurements of $\rho-\omega$ interference in the reaction $\pi^{-} p \rightarrow \pi^{+} \pi^{-} n$ at $15 \mathrm{GeV} / \mathrm{c}$, Phys.Lett. 38B (1972) 345

[7] M. L. Stevensson et al., Spin and parity of the $\omega$ meson, Phys.Rev. 125 (1962) 687

[8] F. Niecknig et al., Dispersive analysis of $\omega \rightarrow 3 \pi$ and $\phi \rightarrow 3 \pi$ decays, Eur.Phys.J. C72 (2012) 2014 [hep-ph/1203.2501]

[9] I. V. Danilkin et al., Dispersive analysis of $\omega / \phi \rightarrow 3 \pi, \pi \gamma *$, [hep-ph/1409 . 7708]

[10] C. Terschlüsen, Reactions with pions and vector mesons in the sector of odd intrinsic parity, Eur.Phys.J. A49 (2013) 116 [hep-ph/1305.1181] 